\title{
INFLUENCE OF IMPACT IONIZATION OF SHALLOW DONORS ON LUMINESCENCE IN GaAs*
}

\author{
A. WYSMOLEK AND J. LUSAKOWSKI \\ Institute of Experimental Physics, University of Warsaw \\ Hoża 69, 00-681 Warszawa, Poland
}

\begin{abstract}
Luminescence spectra of $n$-type molecular beam epitaxial layer and semi-insulating liquid encapsulated Czochralski grown bulk GaAs were measured at liquid helium temperature for zero electric field and for fields which caused impact ionization of shallow donors. Application of the electric field caused a decrease in the luminescence intensity and a broadening of all observed structures. It was found that the electric field changed the luminescence spectrum of the $n$-type material in a different way than it did in the case of the semi-insulating one. For the $n$-type sample, an intensity of excitonic lines decreased much more than that of donor-acceptor lines when the electric field grew. A contrary was observed for the semi-insulating sample. An explanation of the result is proposed which takes into account an influence of ionised impurity scattering and localization in fluctuations of the electrostatic potential on the luminescence process.
\end{abstract}

PACS numbers: $72.20 . \mathrm{Ht}, 78.55 .-\mathrm{m}$

\section{Introduction}

Long range fluctuations of the electrostatic potential arise in compensated semiconductors due to a random spatial distribution of charged carriers [1]. An amplitude of fluctuations grows with an increase in a concentration of these charges which are responsible for creating a fluctuating potential, and decrease with an increase in a concentration of screening charges. Thus, in compensated semiconductors at low temperatures, an amplitude of fluctuations is greater than in low doped high quality materials.

An influence of potential fluctuations on magneto-optical and magnetotransport properties of semi-insulating (SI) GaAs was recently investigated [2-5]. A part of that study was devoted to an analysis of the conductivity of hot electrons at

*This work is partially supported by the State Committee for Scientific Research (Republic of Poland) grants No. 202659101 and PBZ-101-01. 
high magnetic fields at liquid helium temperatures [2-4]. The electron gas was then heated simultaneously by optical and electric field excitations. The optical excitation was achieved by illuminating a sample with a near-band light, which excited electrons to the conduction band, otherwise empty at low temperatures. Excited electrons thermalized into wells of the fluctuating potential at the bottom of the conduction band and some of them were captured by shallow donors [5]. A sufficiently high electric field caused impact ionization of shallow states at the bottom of the conduction band (neutral donors and wells of the fluctuating potential), which resulted in a jump of the current by a few orders of magnitude. Impact ionization of shallow donors was also observed for a $n$-type MBE layer of GaAs at voltages essentially lower than that for SI samples.

Experimental conditions required for investigation of a hot electron transport in SI GaAs, i.e. a permanent illumination and high electric fields, are also adequate for studying an influence of impact ionization of shallow donors on the near-band luminescence in this material. Such studies were performed by others on $n$-type GaAs samples and described in a number of papers [6-9]. The aim of the present study is to compare an influence of impact ionization on luminescence in $n$-type and SI GaAs.

\section{Experiment and discussion}

Luminescence spectra were measured at $4.2 \mathrm{~K}$ in the energy range from $1.48 \mathrm{eV}$ to $1.52 \mathrm{eV}$ with $1.85 \mathrm{eV}$ diode laser excitation for SI GaAs and $n$-GaAs samples. The exciting light power was equal to about $2 \mathrm{~mW}$. The SI sample was an undoped LEC crystal with EL2 as the main deep level with its concentration equal to $5.3 \times 10^{16} \mathrm{~cm}^{-3}$ and the $n$-type sample was characterised by a shallow donors concentration and a compensation equal to about $10^{14} \mathrm{~cm}^{-3}$ and 0.2 , respectively. Measurements were performed after bleaching EL2 to its metastable state. An avalanche breakdown was observed for about $0.2 \mathrm{~V}$ and for about $30 \mathrm{~V}$ of a bias applied to the $n$-type and SI sample, respectively.

Results of measurements are shown in Fig. 1 and in Fig. 2 for the $n$-type and the SI sample, respectively. The spectra shown in Fig. 1 and in Fig. 2 are normalised at the donor-acceptor peak. The spectrum of the $n$-type GaAs is dominated by the excitonic lines, whereas that of the SI sample shows band-acceptor and donor-acceptor lines as the dominant structures. For both materials, in the impact ionization regime the intensity of luminescence decreased, and the width of the monitored structures increased. For the SI sample, an amplitude of band-acceptor and donor-acceptor structures decreased more than that of an excitonic one. An opposite effect was observed for the case of the $n$-type sample, for which the electric field influenced much more the excitonic lines. This can be deduced by comparing a sequence of curves in Fig. 1 and Fig. 2 at excitonic energies. No measurable shift of positions of donor-acceptor and conduction band-acceptor lines was observed which shows that an influence of a bolometric effect on the luminescence intensity can be neglected [10].

The observed decrease in luminescence intensity with an increase in the electric field is consistent with literature data [8, 9]. From Fig. 1 and Fig. 2 one 


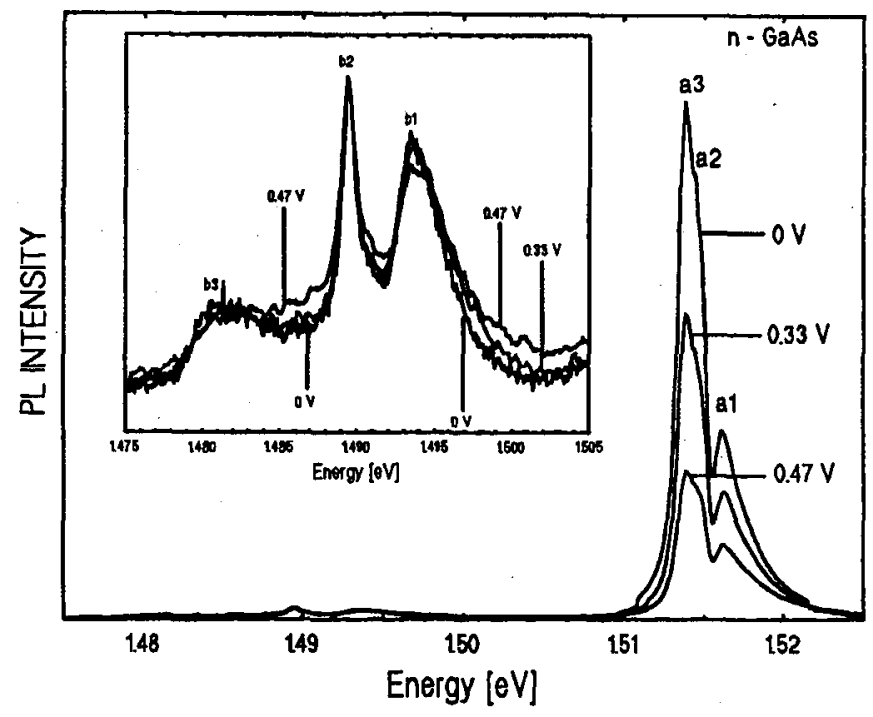

Fig. 1. Luminescence spectra for $n$-type GaAs at $T=4.2 \mathrm{~K}$ for indicated voltages applied to the sample. The $b 1, b 2$ and $b 3$ peaks correspond to conduction band-acceptor (C), donor-acceptor and conduction band-acceptor ( $\mathrm{Si}$ transitions, respectively. The $a 1, a 2$ and $a 3$ lines correspond to a luminescence of a free exciton, an exciton bound on a donor and an acceptor, respectively.

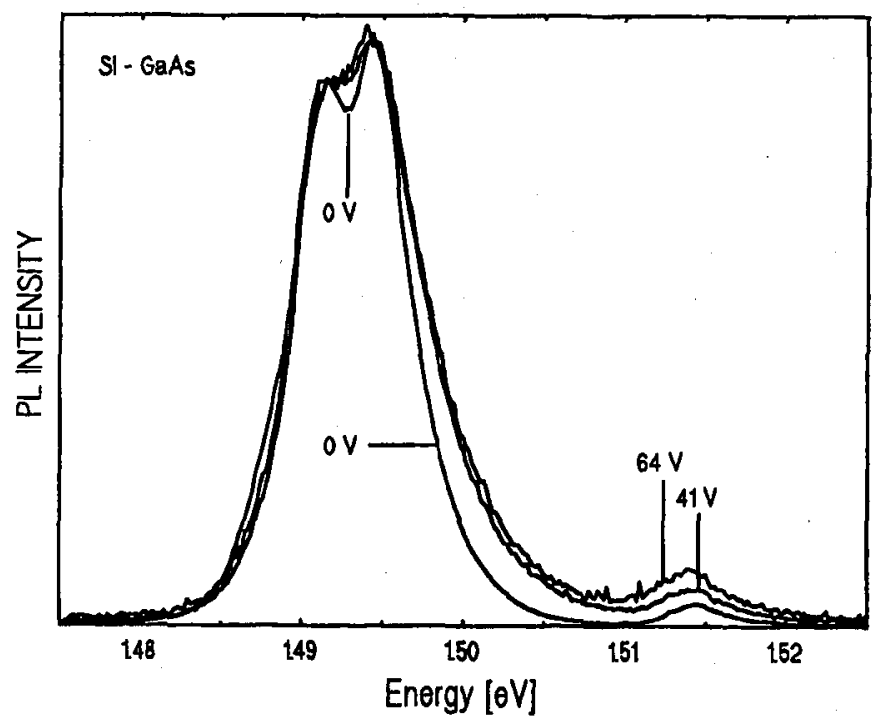

Fig. 2. Luminescence spectra for SI GaAs at $T=4.2 \mathrm{~K}$ for indicated voltages applied to the sample. The high-energy structure comes from exciton luminescence and the low-energy one form that of conduction band-acceptor (the highest peak) and of donor-acceptor. 
can conclude that an influence of the electric field on the low-energy band of a luminescence spectrum is similar for $n$-type and SI sample. Ilowever, a clear difference between the two materials is observed at the high-energy band of the spectrum. To discuss this difference one should notice that the mechanism which decreases an intensity of exciton luminescence at high electric fields is impact ionization of excitons [11]. Thus, an influence of the electric field on effectiveness of this process determines a rate of decrease in exciton luminescence.

An ability of the electric field to heat an electron gas grows with decreasing the rate of electron scattering. At liquid helium temperatures the dominant scattering is by ionised impurities. The electron mean free path in $n$-type material is longer than in SI one, which leads to a smaller electron mobility in SI than in $n$-type GaAs. This causes a smaller influence of an applied electric field on the rate of impact processes in the SI material. A further reduction of an influence of the electric field on an electron motion in SI GaAs comes from a tendency of electrons to localise in wells of a fluctuating potential, which limits a number of free electrons capable to impact ionise.

In conclusion, scattering of free carriers by ionised impurities and electron localization in fluctuations of the electrostatic potential are proposed to be responsible for different influence of an external electric field on exciton luminescence in $n$-type and SI-GaAs.

\section{Acknowledgments}

The authors are thankful to Dr. E. Kamińska and Dr. A. Piotrowska, Institute of Electron Technology, Warsaw, for preparing contacts to the samples.

\section{References}

[1] B.I. Shklovskii, A.L. Efros, Electronic Properties of Doped Semiconductors, Springer-Verlag, Berlin 1984.

[2] J. Łusakowski, R. Merten, M. Grynberg, Acta Phys. Pol. A 82, 551 (1992).

[3] J. Eusakowski, K. Karpierz, M.L. Sadowski, M. Grynberg, Solid State Commun. 84, 403 (1993).

[4] K. Karpierz, J. Eusakowski, M.L. Sadowski, M. Grynberg, Physica $B$ 184, 403 (1993).

[5] M.L. Sadowski, K. Karpierz, M. Grynberg, Phys. Rev. B 34, 7332 (1991).

[6] W. Schairer, N. Stath, J. Appl. Phys. 43, 447 (1972).

[7] W. Bludau, E. Wagner, Appl. Phys. Lett. 29, 204 (1976).

[8] M. Yamawaki, C. Hamaguchi, Phys. Status Solidi B 112, 201 (1982).

[9] F. Karel, J. Oswald, J. Pastrnak, O. Petricek, Semicond. Sci. Technol. 7, 203 (1992).

[10] R. Dingle, Phys. Rev. 184, 788 (1969).

[11] B.J. Skromme, G.E. Stillman, Phys. Rev. B 28, 4602 (1983). 